\title{
Depletion of Thymosin $\beta 4$ Promotes the Proliferation, Migration, and Activation of Human Hepatic Stellate Cells
}

\author{
Yongtao Xiao ${ }^{b, c}$ Chunying $\mathrm{Qu}^{\mathrm{a}}$ Wensong Ge $\mathrm{G}^{\mathrm{a}}$ Baocan Wang ${ }^{\mathrm{a}}$ Jianxin $\mathrm{Wu}^{\mathrm{a}}$ \\ Leiming $\mathrm{Xu}^{\mathrm{a}}$ Yingwei Chen ${ }^{\mathrm{a}, \mathrm{c}}$ \\ aDepartment of Gastroenterology, Xinhua Hospital, School of Medicine, Shanghai Jiao Tong University, \\ bShanghai Institute for Pediatric Research, 'Shanghai Key Laboratory of Pediatric Gastroenterology and \\ Nutrition; Shanghai, China
}

\section{Key Words}

Thymosin $\beta 4$ • Liver fibrosis • Hepatic stellate cell • Proliferation • Migration

\begin{abstract}
Background \& Aims: It has recently been reported that thymosin beta-4 (Tß4) has antifibrogenic effects in human hepatic stellate cells (HSCs) in vitro, but the mechanisms underlying these effects remain unclear. The aim of this study was to investigate the roles of T $\beta 4$ in the proliferation, migration, and activation of HSCs. Methods: Enzyme-linked immunosorbent assays (ELISA), immunohistochemistry, and western blot assays were utilized to determine the expression levels of T $\beta 4$ in serum, liver tissues, and LX-2 cells. T 34 was depleted in LX-2 cells using small interfering RNAs (siRNAs). Cell proliferation was analyzed using cell counting kit-8 (CCK-8) viability assays, and cell migration was investigated using wound-healing and transwell migration assays. Results: The expression of T $\beta 4$ was significantly reduced during the progression of liver fibrosis. The depletion of T $\beta 4$ significantly promoted the proliferation and migration of LX-2 cells via the activation of the PI3K/Akt signaling pathway. The promigratory and pro-proliferative effects of T $\beta 4$ depletion in LX-2 cells can be counteracted by treatment with the Akt inhibitor MK-2206. In addition, TB4 depletion was also associated with the activation of HSCs via the enhanced expression of $\alpha$-smooth muscle actin ( $\alpha-S M A)$ and vimentin. Conclusions: Our results suggest that T $\beta 4$ participates in liver fibrosis by inhibiting the migration, proliferation, and activation of HSCs and that T $\beta 4$ may be an effective target in the treatment of liver fibrosis.
\end{abstract}

Y. Xiao and C. Qu contributed equally to this work. 
Xiao et al.: Thymosin $\beta 4$ was Involved in Liver Fibrosis

\section{Introduction}

Liver fibrosis, a major cause of the morbidity and mortality of chronic liver disease, is characterized by excess synthesis and deposition of extracellular matrix (ECM), which disrupts the normal architecture of the liver and leads to organ dysfunction. Advanced liver fibrosis results in cirrhosis, liver failure, and portal hypertension and often requires liver transplantation [1-3]. Although there are limited therapeutic strategies for treating liver fibrosis, we now have a better understanding of the cellular and molecular mechanisms that underlie this condition. Hepatic stellate cells (HSCs) are the primary cellular source of matrix components in patients with chronic liver disease. Therefore, HSCs play an important role in the development of liver fibrosis [4]. During liver injury, HSCs are activated by cytokines and growth factors such as platelet-derived growth factor (PDGF) and transforming growth factor- $\beta$, which are derived from activated Kupffer cells and damaged hepatocytes. In response to fibrogenic stimuli, HSCs lose their retinoid stores, proliferate, express $\alpha$-smooth muscle actin ( $\alpha$-SMA), and produce large amounts of extracellular matrix proteins including type I collagen [5]. The human HSC cell line LX-2 was used in this study because of its similarity to primary human stellate cells; LX- 2 cells express $\alpha$-SMA, vimentin, platelet derived growth factor receptor $\beta$ (PDGF-R $\beta$ ), matrix metalloproteinase (MMP)2 , and tissue inhibitor of matrix metalloproteinase (TIMP)-2 [6]. PI3K (phosphoinositide 3 -kinase) is composed of an $85-\mathrm{kDa}$ regulatory subunit and a $110-\mathrm{kDa}$ catalytic subunit, which is recruited to and activated by the activated PDGF receptor following HSC activation and growth factor stimulation [7-9]. It has been reported that the activation of the PI3K/ Akt pathway can facilitate HSC proliferation and $\alpha 1$ collagen expression $[7,10,11]$. In this regard, the activation of PI3K/Akt signaling significantly contributes to fibrotic progression.

$\mathrm{T} \beta 4$ is a member of the beta-thymosin family that is expressed in nearly all cell types and has multiple physiological and pathological functions such as wound healing and angiogenesis $[12,13]$. Several studies have suggested that $T \beta 4$ is involved in the process of fibrosis in different organs. T $\beta 4$ has been shown to have pro-fibrotic effects in mice with ureteral obstruction [14]. However, Ac-SDKP, a degradation product of T $\beta 4$, has anti-fibrotic activity [15]. When mediated by elevated expression of hepatocyte grow factor (HGF) and decreased PDGF-dependent phosphorylation, T $\beta 4$ demonstrated anti-fibrogenic effects in hepatic stellate cells (HSCs) in vitro $[10,16]$. Based on these studies, we further investigated whether T $\beta 4$ can suppress the activation of HSCs and examined the molecular mechanisms of $\mathrm{T} \beta 4$ involvement in proliferation, migration, and activation. We first detected the expression of T $\beta 4$ protein in the serum of cirrhosis patients and in liver specimens of bile duct ligation (BDL) rats with liver fibrosis progression. Secondly, we investigated the roles of T $\beta 4$ in the activation of HSCs in terms of proliferation, migration, and ECM deposition. Finally, we studied the role of the PI3K/Akt signaling pathway in LX-2 cells with regard to T $\beta 4$-mediated effects.

\section{Materials and Methods}

\section{Materials and Specimen preparation}

T $\beta 4$ was purchased from Prospec (Rehovot, Israel). Fetal bovine serum (FBS) was purchased from GIBCO (Los Angeles, CA, USA). Anti-Akt, anti-phospho-Akt, anti-Vimentin, anti-phospho-p85, anti-phospho-PDK1, anti-phospho-PTEN, anti-phospho-GSK-3 $\beta$ and anti-GAPDH antibodies were obtained from Cell Signaling Technology (Beverly, MA, USA). The anti-T $\beta 4$ and anti- $\beta$-actin antibodies were obtained from Santa Cruz Biotechnology (Santa Cruz, CA). The Anti-MMP-2 antibody was purchased from Abcam (New Territories, HK). The anti- $\alpha$-SMA antibody was obtained from Sigma-Aldrich (MO, USA). MK-2206 was purchased from Selleck Chemicals (TX, USA). Lipofectamine RNAiMAX was obtained from Invitrogen (CA, USA). Serum was collected from 20 patients with cirrhosis and 15 healthy controls in Xinhua Hospital, Shanghai, China. The serum was obtained between 2010 and 2012 according to informed consent regulations and with the approval of the Ethical Review Committee of Xinhua Hospital. All of the patients provided written informed consent. 
Rat bile duct ligation (BDL)

A total of 30 male Sprague-Dawley rats (200-250 g) underwent bile duct ligation surgery as described previously $[17,18]$ and were sacrificed after 4 or 6 weeks. The animal study was approved by the Animal Care and Use Committee of Xinhua Hospital, Shanghai, China.

Enzyme-linked immunosorbent assays (ELISA)

The T $\beta 4$ ELISA kit was purchased from BlueGene Biotech Co., Ltd. Experiments using this kit were performed according to the protocol described by the manufacturer. The results were determined using a microplate reader at $450 \mathrm{~nm}$ (Molecular Devices, Beckman).

\section{Masson's trichrome staining}

The liver tissues were collected and fixed in $10 \%$ buffered formalin and embedded in paraffin. The samples were sliced into $8 \mu \mathrm{m}$-thick sections. Masson's trichrome staining was performed on the sections according to the protocol described by the manufacturer (GeneMed Scientists Inc). When using this stain, the collagen fibers will be stained blue, the nuclei will be stained black, and the background will be stained red.

\section{Immunohistochemistry and immunofluorescence assays}

Immunohistochemistry was performed with the chromogen diaminobenzidine (DAB) as described previously [19]. Briefly, the samples were incubated with xylol and descending concentrations of ethanol. Endogenous peroxidases were removed by incubation with $0.3 \% \mathrm{H}_{2} \mathrm{O}_{2}$ for $15 \mathrm{~min}$ at room temperature (RT). After antigen retrieval, blocking was performed with using $5 \%$ bovine serum albumin (BSA) for 30 min at RT. An anti-T $\beta 4$ antibody was applied at its optimal concentration overnight in a wet chamber (at a dilution of 1:50). The slides were rinsed in phosphate-buffered saline (PBS) and incubated with the appropriate secondary antibody for $1 \mathrm{~h}$ at RT. Antibody binding was visualized using a liquid DAB Substrate Chromogen System (Dako, Glostrup, Denmark). The slides were rinsed in PBS and counterstained with hematoxylin. For immunofluorescence analysis, the cells were initially fixed with $4 \%$ paraformaldehyde. After blocking the cells with $3 \%$ BSA for 30 min at RT, the cells were incubated with the anti-T $\beta 4$ antibody or anti-MMP-2 antibody (Abcam, Hongkong, Ltd) at RT for 2 h. The cells were washed three times with PBS and then incubated with the secondary antibody conjugated to FITC for $1 \mathrm{~h}$ at RT. The nuclei were counterstained with DAPI. The results were visualized using a fluorescence microscope (ECLIPSE, Nikon).

\section{Cell culture and Transfection}

The human hepatic stellate cell line LX-2 was cultured in Dulbecco's modified Eagle medium (DMEM) supplemented with $10 \%$ fetal bovine serum in a humidified $37^{\circ} \mathrm{C}$ atmosphere of $5 \%$ $\mathrm{CO}_{2}$. The pooled T $\beta 4$ siRNA duplexes were synthesized by Shanghai Genepharma Co., Ltd. The siRNA sequences were 5'-UCGAUAAGUCGAAACUGAATT-3', 5'-CUUCCAAAGAAACGAUUGATT-3' and 5'-GAGGUUGGAUCAAGUUUAATT-3'. The siRNAs were transfected into LX-2 cells with Lipofectamine RNAiMAX.

\section{Western blots}

Western blots were performed according to standard procedures. Briefly, the protein concentrations of the lysates was determined using a bicinchoninic acid protein assay system (Pierce, Rockford, IL), and equal amounts of each sample were separated by electrophoresis on Novex $4-12 \%$ Tris-glycine gels and transferred onto nitrocellulose membranes using a dry blotting system (iBLOT system, Invitrogen, CA, USA). The membrane was pre-incubated for an hour in phosphate-buffered saline (PBS) containing $5 \%$ skim milk and $0.05 \%$ Tween 20 (PBS-T). After blocking, the membranes were incubated with the primary antibodies overnight at $4^{\circ} \mathrm{C}$. The following antibody dilutions were used: Anti-Akt, 1:1,000 dilution; MMP2, 1:1,000 dilution; $\alpha$-SMA, 1:500 dilution; Vimentin, 1:1,000 dilution; p-p85, 1:1,000 dilution; p-PDK1, 1:1,000 dilution; $p$-PTEN, 1:1,000 dilution; p-GSK-3 $\beta$, 1:1,000 dilution; and GAPDH, 1:1,000 dilution. The membranes were washed three times with PBS-T and then incubated with a species-appropriate horseradish peroxidase-conjugated secondary antibody for 1 hour at room temperature. The membranes were washed three times with PBS-T, and the bound antibody was detected with an enhanced chemiluminescence (ECL) detection kit (Thermo Scientific) using a Molecular Imager ChemiDoc ${ }^{\text {TM }}$ XRS (Bio-RAD). 


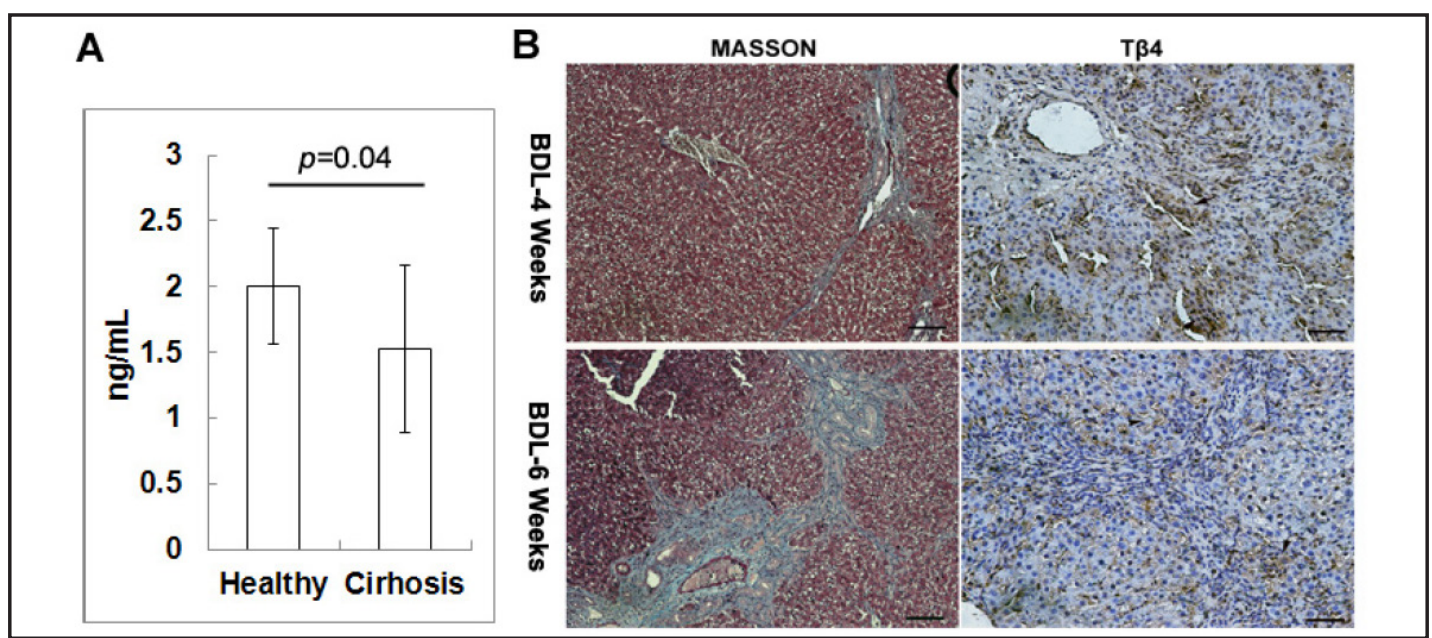

Fig. 1. The expression of T $\beta 4$ decreases in liver fibrosis. (A) The serum levels of $\mathrm{T} \beta 4$ were significantly lower in patients with cirrhosis compared to healthy controls. (B) The expression of T $\beta 4$ in rat liver was significantly lower 4 to 6 weeks after BDL and correlated with the progression of fibrosis.

\section{Wound-healing and Transwell migration assays}

Confluent cells in six-well plates were scraped using a pipette tip to make a 22 mm-long wound. The cells were incubated at $37^{\circ} \mathrm{C}$ and allowed to migrate into the wound and then fixed. After fixation, the numbers of cells that moved into the scratched area were counted in a microscope. The migration ratio (\%) was calculated by the width of the wound at 16 and 24 hours divided by the width of the wound at 0 h. Cell migration was evaluated in 24-well transwell chambers (Corning, MA, USA), as described by the manufacturer. Briefly, the lower chambers of the 24-well plate were filled with $500 \mu$ l of DMEM containing $10 \%$ FBS. A suspension of $1 \times 10^{4}$ cells in $100 \mu$ l of DMEM without FBS was placed into the upper compartments of the wells. The transwell chambers were incubated at $37^{\circ} \mathrm{C}$ in a humidified $5 \% \mathrm{CO}_{2}$ atmosphere for $24 \mathrm{~h}$. The cells that had invaded the lower surface of the polycarbonate membranes (12 $\mu \mathrm{m}$ pore size) were fixed, stained with Coomassie blue, and quantified by counting 3 microscopic fields for each filter.

\section{Co-immunoprecipitation}

LX-2 lysates were precipitated with T $\beta 4$ antibody using a Co-IP kit (Pierce, IL USA) as previously described [20]. Western blots were performed using an anti-actin polyclonal antibody (Santa Cruz Biotechnology).

Cell viability assay

A total of $3 \times 10^{3}$ cells/well was seeded in 96-well plates 24 hours prior to treatment. The proliferation of the treated cells was determined by using Cell Counting Kit- 8 assays (Dojindo, Kumamoto, Japan). The absorbance value of each well was determined at $450 \mathrm{~nm}$ using a microplate reader (Molecular Devices, Beckman).

\section{Statistical Analysis}

All data are reported as the mean \pm S.D. Statistical significance was determined by Student's t-tests using the SPSS19 software program when comparisons were made between two different groups.

\section{Results}

T $\beta 4$ is down-regulated with liver fibrosis progression

A previous study has evidenced that hepatocytes were able to synthesize large amounts of T $\beta 4$. Furthermore, a strong diffuse expression of T $\beta 4$ was observed in the human liver [21]. However, the intracellular and extracellular roles played by T $\beta 4$ under physiological conditions or in the context of different liver diseases are still unclear. In this study, we first 
Fig. 2. T $\beta 4$ depletion promotes the proliferation of LX-2 cells and activated the PI3K/Akt signaling pathway. (A) T $\beta 4$ significantly inhibited the growth of LX-2 cells and markedly suppressed the phosphorylation of Akt (Ser 473 and Thr 308). (B) Immunofluorescence analysis showed that $\mathrm{T} \beta 4$ protein was expressed strongly in LX-2 cells. (C) T $\beta 4$ depletion induced the phosphorylation of Akt and significantly increased the proliferation of LX-2 cells. (D)T $\beta 4$ depletion activated the PI3K signaling pathway by affecting the phosphorylation of selected components. T $\beta 4$ depletion increased phosphorylation of p85 (Tyr 458), PDK1 (Ser 241), and GSK-3 $\beta$ (Ser 9), but inhibited the phosphorylation of PTEN. Magnified 200×.
A
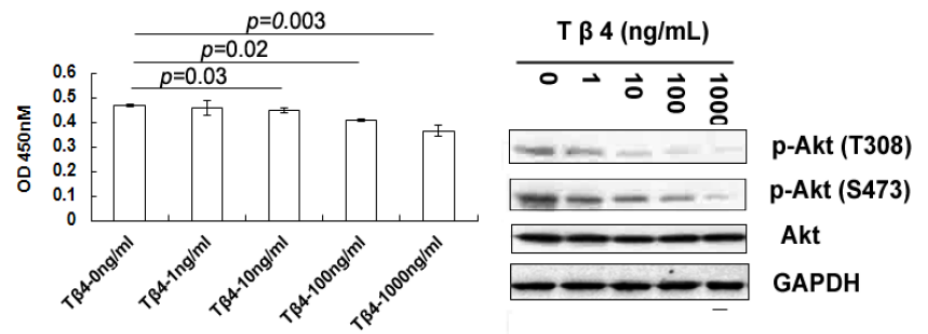

B

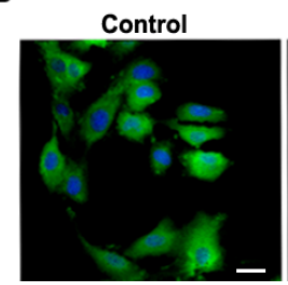

TB4-SiRNA

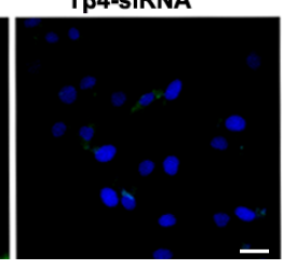

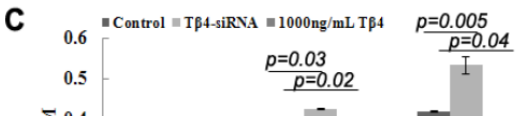

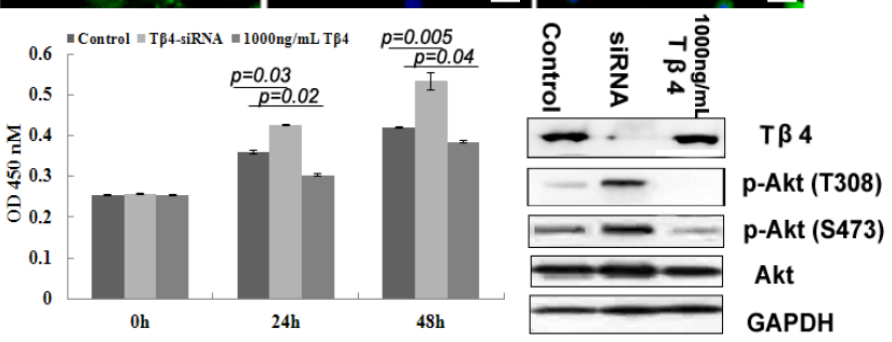

D
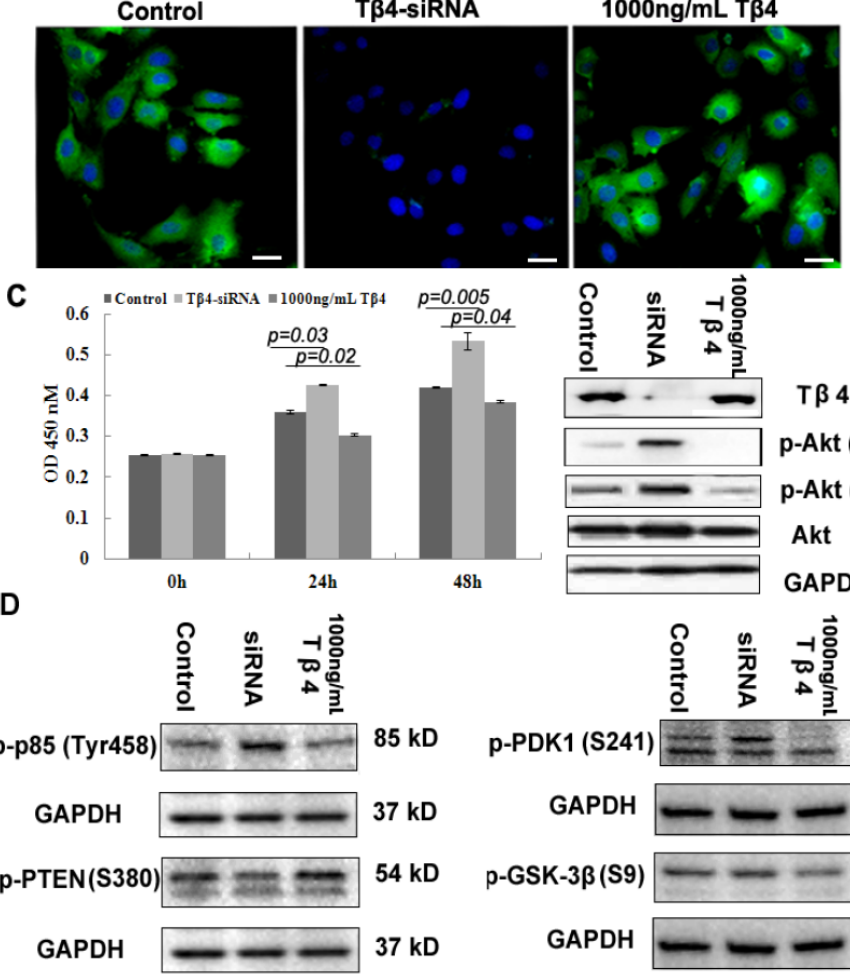

p-PDK1 (S241) $=58$ = $58,68 \mathrm{kD}$

GAPDH

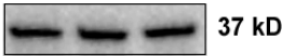

p-GSK-3 $\beta$ (S9)

$---46 \mathrm{kD}$

GAPDH

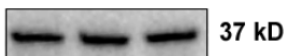

examined the expression of T $\beta 4$ in patients with cirrhosis and rats that underwent BDL. We compared the serum expression levels of T $\beta 4$ in 17 patients with cirrhosis and 15 healthy controls. Serum T $\beta 4$ levels were significantly lower in cirrhosis patients $(1.5 \pm 0.5$ $\mathrm{ng} / \mathrm{mL})$ than in healthy controls $(2.1 \pm 0.4 \mathrm{ng} / \mathrm{mL})(\mathrm{p}=0.04)$ (Fig. 1A). We further used immunohistochemistry analysis to investigate the expression of $\mathrm{T} \beta 4$ in rat liver specimens from the BDL model. The degrees of rat liver fibrosis were determined by Masson's trichrome staining after the rats were sacrificed at 4 and 6 weeks. The levels of T $\beta 4$ were significantly lower 4 and 6 weeks after BDL and were negatively correlated with the progression of fibrosis progression (Fig. 1B).

\section{T $\beta 4$ depletion increased the proliferation of LX-2 cells}

Because the down-regulation of serum $\mathrm{T} \beta 4$ was observed in patients with cirrhosis, we further investigated whether $\mathrm{T} \beta 4$ has biological anti-fibrosis properties. The human HSCs cell line LX-2 has been extensively used as a tool for studying human hepatic fibrosis since its establishment in 2005 [6]. In this study, we investigated whether T $\beta 4$ had any effect on the proliferation of LX- 2 cells. LX- 2 cells were incubated with different concentrations of T $\beta 4(0$, 10, 100, and 1,000 ng/mL) for 24 hours. As illustrated in Fig. 2A, the growth of LX-2 cells was significantly inhibited after T $\beta 4$ treatment. Furthermore, the proliferation of LX- 2 cells was significantly enhanced when T $\beta 4$ was knocked down with siRNA (Fig. 2B, C). 
Fig. 3. T $\beta 4$ depletion promotes the proliferation of LX-2 cells by activating Akt. (A)LX-2 cells acquired neural-like morphologies after treatment with the Akt inhibitor MK-2206. (B) Proliferative analysis indicated that the proliferative effect mediated by $\mathrm{T} \beta 4$ depletion was significantly attenuated by MK-2206. Magnified 200×.

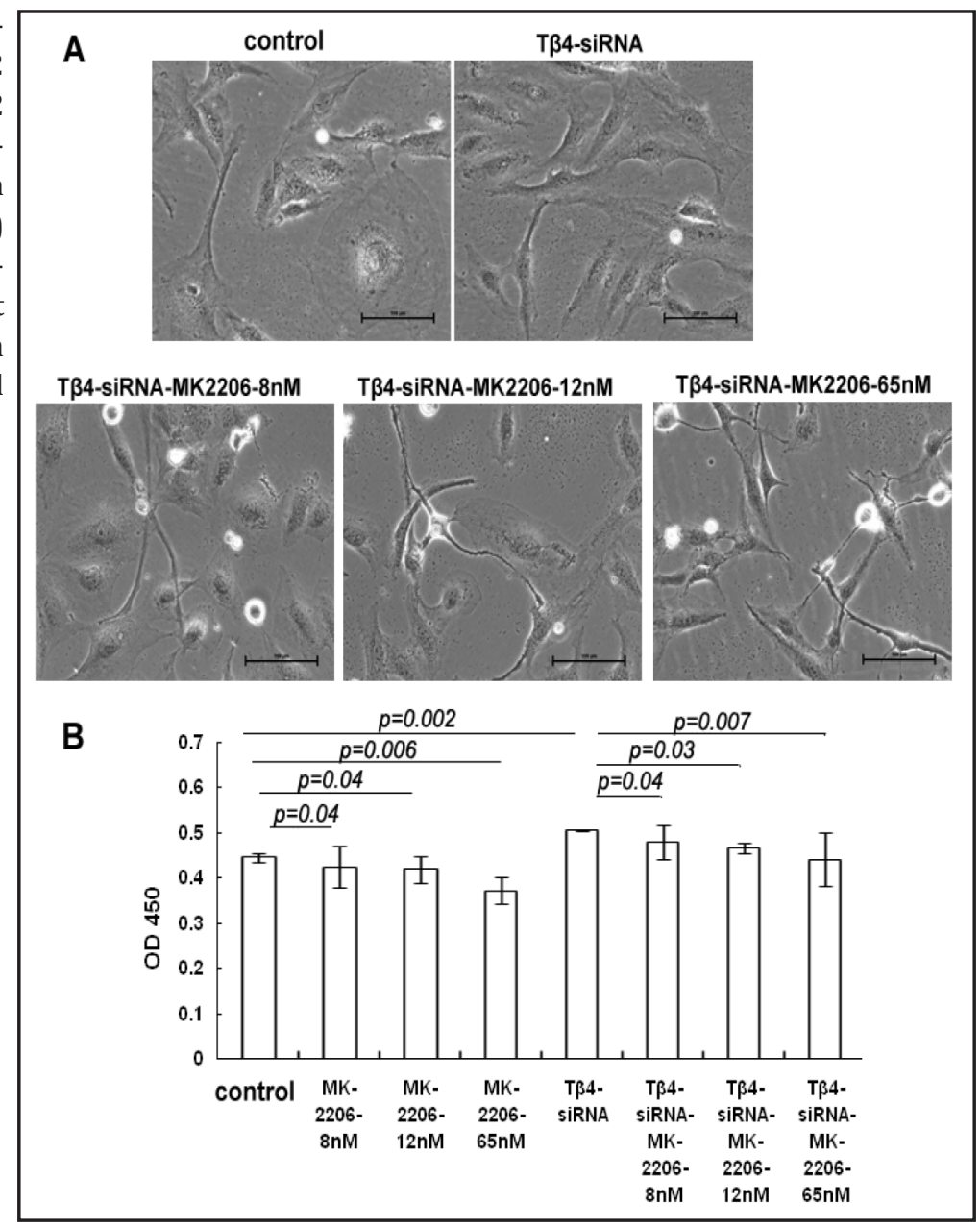

T $\beta 4$ depletion activated the PI3K/Akt signaling pathway in LX-2 cells

The phosphorylation of Akt (Ser 473 and Thr 308) can be inhibited by T $\beta 4$ treatment. Correspondingly, T $\beta 4$ depletion can significantly induce the phosphorylation of Akt at both sites (Fig. 2A-C). Because Akt is a downstream factor in the PI3K/Akt signaling pathway, further investigation is necessary to determine whether T $\beta 4$ has an effect on the degree of phosphorylation of the components in this pathway. As illustrated in Fig. 2D, T $\beta 4$ depletion increased phosphorylation of the regulatory subunit of PI3K, p85 (Tyr 458), and the kinase PDK1 (Ser 241). We further analyzed the expression and phosphorylation of PTEN, the phosphatase that prevents PI3K-mediated effects by hydrolyzing phosphatidylinositol $(3,4,5)$-trisphosphate to phosphatidylinositol 4, 5-bisphosphate; we observed that the phosphorylation of PTEN (Ser 380) was inhibited by T $\beta 4$ depletion. Glycogen synthase kinase-3 $\beta$ (GSK-3 $\beta$ ) is a well-established downstream component of the phosphatidylinositol 3-kinase (PI3K) signaling pathway. Fig. 2D shows that the phosphorylation of GSK-3 $\beta$ (Ser 9) was enhanced by T $\beta 4$ depletion. To investigate whether Akt activation is essential to the T $\beta 4$-mediated proliferative effect in LX-2 cells, LX-2 cells were treated with the Akt inhibitor MK-2006 (at concentrations of 8, 12, and $65 \mathrm{nM}$ ) for 24 hours prior to T $\beta 4$ siRNA transfection and examined by morphological observation and proliferative assays [22]. The growth of LX-2 cells was significantly promoted by T $\beta 4$ depletion compared to control $(p=0.002)$. MK-2206 treatment decreased the viability of LX-2 cells and was accompanied by the appearance and outgrowth of neurite-like structures (Fig. 3). 
Fig. 4. $T \beta 4$ depletion enhanced the migration of LX-2 cells. Wound-healing and Transwell migration assays showed that treatment with $\mathrm{T} \beta 4$ peptide suppressed LX-2 cell migration, while T $\beta 4$ depletion induced cell migration. (A, B)Woundhealing assays. (C) Transwell analysis. $*, \mathrm{p}<0.05 ;{ }^{* *}, \mathrm{p}<0.01$, magnified $40 x$.

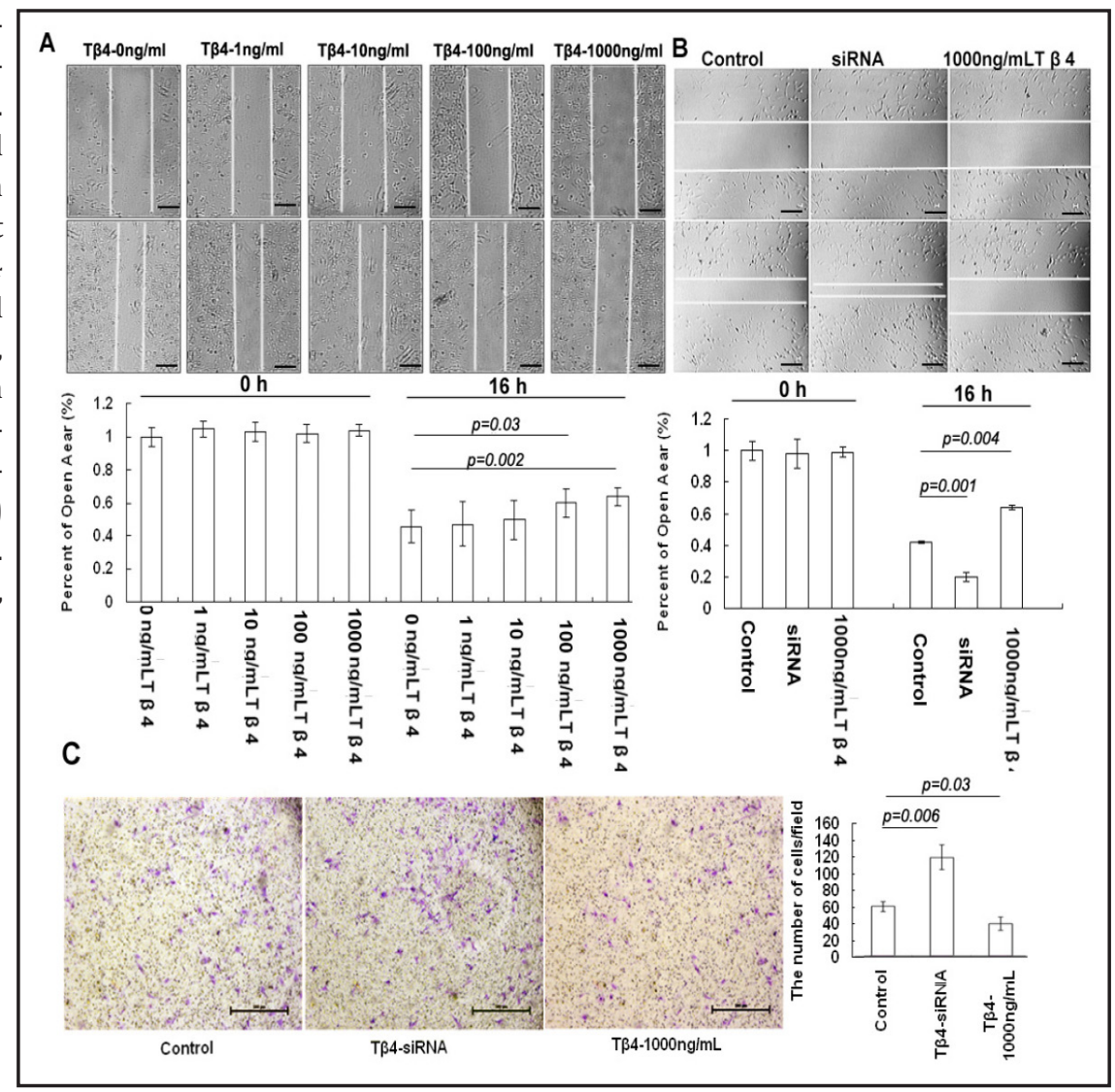

Tß4 depletion promoted the migration of LX-2 cells

In addition, we utilized scratch-wound healing and Transwell migration analysis to examine the effects of T $\beta 4$ on the migration of LX-2 cells. As shown in Fig. 4A, wound-healing assays showed that approximately $38 \%$ of the wounds remained open after 16 hours. The wounds that were treated with $\mathrm{T} \beta 4$ were significantly wider than the untreated controls. In contrast, after T $\beta 4$ depletion, more LX-2 cells migrated into the wound area, and only $20 \%$ of the wounds remained open (Fig. 4B). For Transwell migration analysis, LX-2 cells were cultured on the $12-\mu \mathrm{m}$ pore size transwell inserts. The serum in the lower chamber served as the chemoattractant, and the cells that migrated through the inserts were counted. As seen in Fig. 4C, T $\beta 4$ depletion resulted in a significant increase in the number of cells that migrated toward the serum, whereas treating the cells with $1,000 \mathrm{ng} / \mathrm{mL} \mathrm{T} \beta 4$ decreased the number of cells that migrated compared to the control.

The mechanisms involved in T $\beta 4$-medicated LX-2 cell migration

$\mathrm{T} \beta 4$ is believed to have an anti-migratory function because it can inhibit actin polymerization in vitro and in vivo and induce cytoskeletal disorganization [23, 24]. Here, we investigated whether T $\beta 4$ can inhibit the migration of LX-2 cells by binding to actin and suppressing actin polymerization. We first used confocal immunofluorescence microscopy with cells stained for actin (red) and T $\beta 4$ (green) to determine whether these two proteins are co-localized in LX-2 cells. As shown in Fig. 5A, T 34 was distributed around the nucleus in untreated cells. In LX-2 cells treated with T $\beta 4$ siRNA, actin polymerization was evident around the cell membrane (Fig. 5A). To confirm the confocal microscopy findings, we immunoprecipitated actin using an antibody and determined the presence of T $\beta 4$ in the immunoprecipitate analysis. As shown in Fig. 5B, actin was indeed co-immunoprecipitated with T $\beta 4$ in LX-2 cells.

In addition to affecting actin polymerization, the dissociation of the actin-T $\beta 4$ complex may influence metalloproteinase synthesis, which is necessary for extracellular matrix 

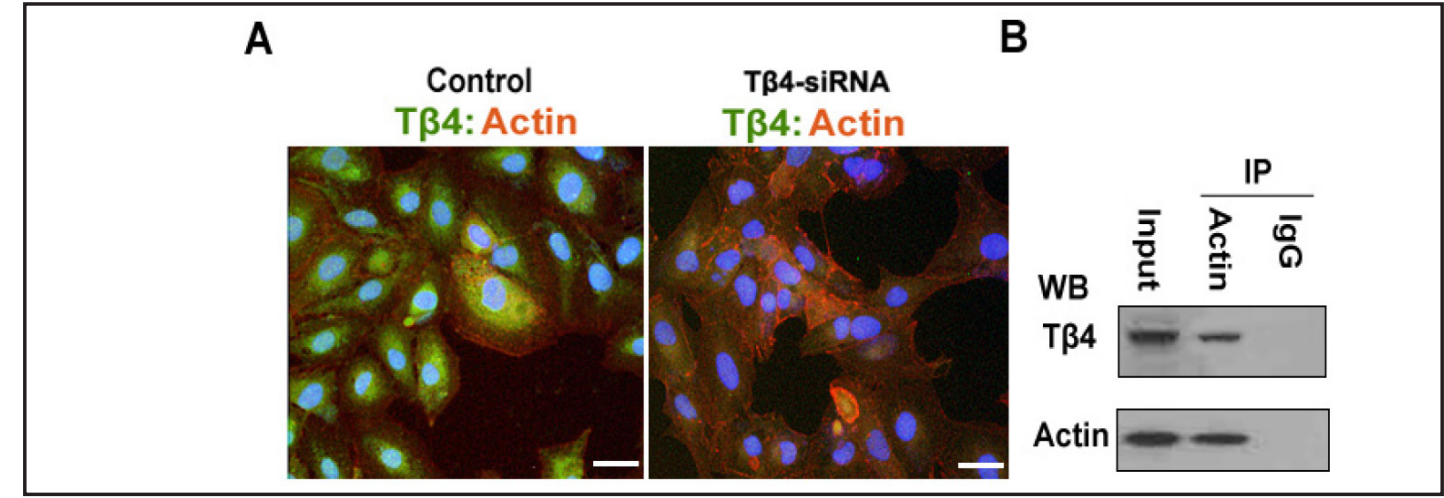

B

Fig. 5. Actin co-immunoprecipitated with T $\beta 4$ in LX-2 cells. (A) Immunofluorescence analysis showed that T $\beta 4$ co-localized with actin in LX-2 cells. The cells were stained with anti-T $\beta 4$ and detected with a greenfluorescent AlexaFluor 488 secondary antibody (Life Technologies). Actin was labeled with AlexaFluor 647 Phalloidin (Life Technologies), and the nuclei were stained with blue-fluorescent DAPI. (B) T $\beta 4$ was immunoprecipitated using an anti-T $\beta 4$ antibody, and the presence of actin in the immunoprecipitate was determined by western blot analysis. Magnified 200x.

Fig. 6. T $\beta 4$ depletion enhanced the migration of LX-2 cells, and this effect was partially dependent on Akt phosphorylation. (A, B)Wound-healing assays showed that the Akt inhibitor MK-2206 inhibited the pro-migratory effect mediated by T $\beta 4$ depletion. (C, D) $\mathrm{T} \beta 4$ depletion induced the expression of MMP-2, but this was counteracted by MK-2206 treatment. Akt2 knockout increased the migration of LX-2 cells. (E) Magnified $40 \times$.

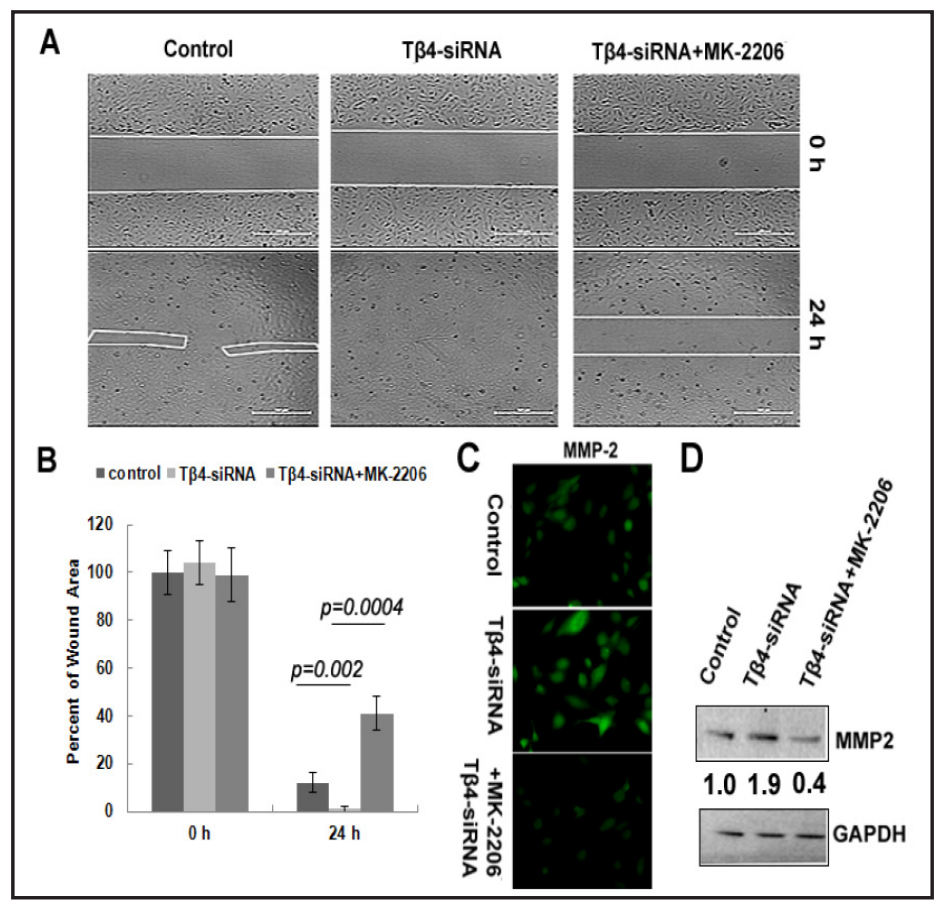

degradation and cell migration [25]. In this study, T $\beta 4$ depletion in LX-2 cells significantly increased cell migration and matrix metalloproteinase (MMP)-2 expression (Fig. 6C, D). We further examined whether Akt is required for the T $\beta 4$ suppression-mediated MMP-2 expression and cell migration. As shown in Fig. 6, the cell migration that was induced by T $\beta 4$ depletion was suppressed by treatment with MK-2206 (Fig. 6A, B).

T $\beta 4$ inhibits $L X-2$ cell activation as determined by the expression of $\alpha-S M A$ and vimentin

$\mathrm{T} \beta 4$ has been reported to counteract fibrosis in cystic fibrosis and lung fibrosis $[25,26]$. In this study, the anti-fibrogenic property of T $\beta 4$ was investigated by examining its effect on HSC activation. LX-2 cells were treated with $1,10,100,1000 \mathrm{ng} / \mathrm{mL}$ of T $\beta 4$ or transfected with T $\beta 4$-siRNA and then incubated for 24 hours. As illustrated in Fig. 7, T $\beta 4$ significantly inhibits the expression of $\alpha$-SMA and vimentin. In contrast, T $\beta 4$ depletion induced the expression of 
Fig. 7. $\mathrm{T} \beta 4$ depletion activates LX-2 cells. T $\beta 4$ knockdown in LX-2 cells increased expression of $\alpha$-SMA and vimentin. In contrast, $\mathrm{T} \beta 4$ treatment suppressed the expression of $\alpha$-SMA and vimentin.

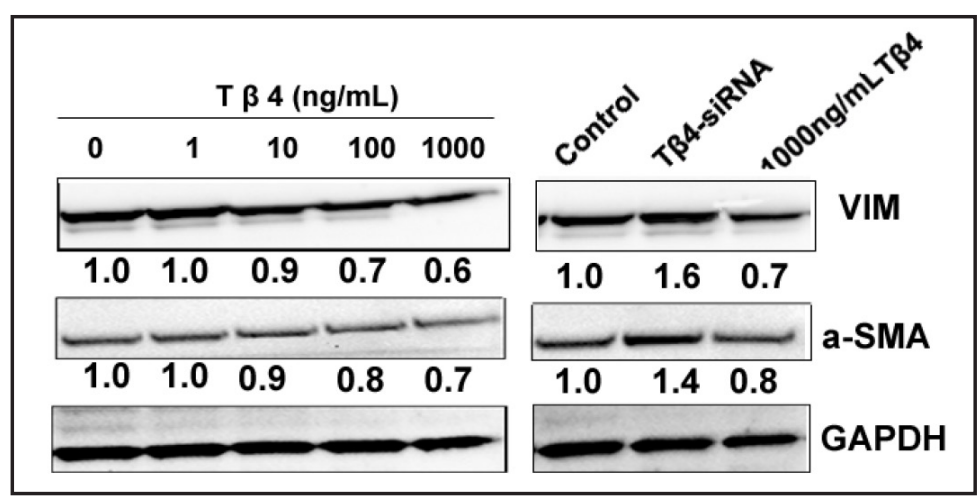

Fig. 8. Possible mechanisms involved in the $\mathrm{T} \beta 4$ depletionmediated proliferation and migration of LX- 2 cells. T $\beta 4$ depletion in LX-2 cells promotes the phosphorylation of Akt, thereby inducing the migration and proliferation of LX-2 cells. In addition, T $\beta 4$ depletion causes disassociation from actin, increases actin polymerization, and then enhances LX-2 cell migration.

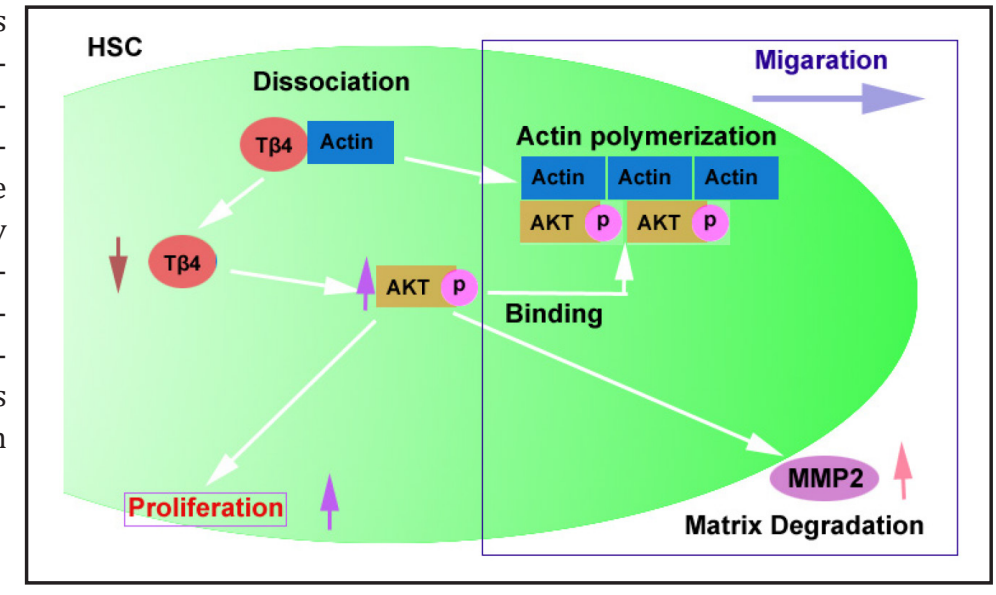

both proteins, suggesting that $\mathrm{T} \beta 4$ can inhibit the activation of $\mathrm{LX}-2$ cells by suppressing the expression of $\alpha$-SMA and vimentin.

\section{Discussion}

$\mathrm{T} \beta 4$, a primary sequestering protein of cellular actin, is involved in many critical biological processes including angiogenesis, wound healing, inflammatory response, and cell migration [13, 27-29]. T $\beta 4$ has been found both inside the cells and in extracellular fluids, such as blood serum, saliva, and wound fluid [30-32]. Nemolato et al. recently reported that hepatocytes were able to synthesize large amounts of T $\beta 4$, but the intracellular roles of T $\beta 4$ were still unclear [21]. In this study, we found that T $\beta 4$ levels were decreased significantly in both human serum and liver tissues during liver the progression of fibrosis. However, the primary mechanism of T $\beta 4$ in liver fibrosis remains to be fully established. HSCs, a key contributor to liver fibrosis, express HGF and its receptor c-Met [33]. Several studies have reported that HGF could ameliorate liver cirrhosis and resolve fibrotic process. This effect is partly due to the suppression of HSC proliferation and the induction of apoptosis in activated cells [34]. A recent study revealed that HGF can induce the expression of T $\beta 4$, which downregulated the expression of the PDGF- $\beta$ receptor and thus prevented the proliferation of HSCs. T $\beta 4$ can also upregulate the expression of HGF, thus establishing a paracrine loop [10, $16]$.

Recent studies showed that T $\beta 4$ upregulates the expression of HGF, which can ameliorate liver cirrhosis and resolve fibrotic progression by suppressing HSC proliferation and inducing apoptosis in activated HSCs. In addition, the HGF-induced expression of T $\beta 4$ can establish a paracrine loop $[10,16,33,34]$. Here, we demonstrated that LX-2 cells strongly expressed T $\beta 4$ and that T $\beta 4$ depletion significantly promoted the growth of LX-2 cells by activating the PI3K/Akt signaling pathway. Akt (serine/threonine protein kinase) is a key 
kinase that regulates multiple cellular processes; it is therefore an ideal target for preventing the activation/transdifferentiation of HSCs. We found that the expression of phosphorylated Akt (Ser 473 and Thr 308) was significantly inhibited by T $\beta 4$ administration. In addition, the Akt inhibitor MK-2206 suppressed Akt phosphorylation and thus counteracted the proliferative effects of T $\beta 4$ depletion on LX-2 cells. Thus, T $\beta 4$ may suppress the activation of HSCs by increasing HGF expression and disrupting PDGF/PI3K/Akt signaling. Other investigators have suggested that oxidative stress is implicated as an important molecular mechanism underlying fibrosis in the liver $[35,36]$. The oxidative stress either contributes to the activation of HSCs and collagen production or stimulates hepatocyte regeneration after the administration of HGF[11, 37]. T $\beta 4$ treatment can reduce the expression of several profibrotic genes, including the connective tissue growth factor (CTGF), collagen type-1 (Col-I), and collagen type-3 (Col-III) in cardiac fibroblasts. [38] However, whether T $\beta 4$ could prevent oxidative stress-induced HSC activation still requires further investigation. In this regard, T $\beta 4$ inhibits HSC activation primarily by inducing HGF expression and disrupting PDGF/ PI3K/Akt signaling.

Locomotion for adherent cells is a highly integrated process that is initiated by the forward extension of lamellipodia and proceeded by repeated cycles of protrusion, adhesion, and contraction $[39,40]$. T $\beta 4$ is a candidate regulator of cell protrusion with important roles in distinct protrusion-related processes such as actin polymerization and matrix metalloproteinase (MMP) expression. Interestingly, T $\beta 4$ apparently has opposing roles in these processes. As a major sequestering protein of globular actin (G-actin), T $\beta 4$ is believed to have an anti-migratory function because it inhibits actin polymerization in vitro and in vivo and induces cytoskeletal disorganization [23, 24]. In contrast, the activation of Akt by T $\beta 4$ is considered to be pro-migratory $[13,41]$. In this study, it was observed that T $\beta 4$ depletion resulted in increased actin polymerization, thereby inducing LX-2 cell migration. In liver fibrosis, activated HSCs produce ECM, which provides cells with positional information and a mechanical scaffold for adhesion and migration by allowing binding to certain growth factors/ cytokines, MMPs, and processing enzymes [42]. To evaluate the influence of Akt activation on HSCs migration that is mediated by T $\beta 4$ depletion, the Akt inhibitor MK-2206 was used in this study. After MK-2206 treatment, the pro-migratory effect induced by T $\beta 4$ depletion was significantly suppressed. Finally, we showed that T $\beta 4$ administration could also suppress the expression of pro-fibrotic proteins, including $\alpha$-SMA and vimentin. Altogether, our results suggested that T $\beta 4$ was a potential anti-fibrogenic agent for the treatment of liver cirrhosis.

\section{Disclosure Statement}

The authors declare no conflicts of interest.

\section{Acknowledgements}

This study is supported by the Shanghai Key Laboratory of Pediatric Gastroenterology and Nutrition (11DZ2260500) and the Shanghai Committee of Science and Technology (11ZR1424000).

\section{References}

1 Moreira RK: Hepatic stellate cells and liver fibrosis. Arch Pathol Lab Med 2007;131:1728-1734.

-2 Dooley S, Hamzavi J, Breitkopf K, Wiercinska E, Said HM, Lorenzen J, Ten Dijke P, Gressner AM: Smad7 prevents activation of hepatic stellate cells and liver fibrosis in rats. Gastroenterology 2003;125:178-191. 
Xiao et al.: Thymosin $\beta 4$ was Involved in Liver Fibrosis

3 Bataller R, Brenner DA: Hepatic stellate cells as a target for the treatment of liver fibrosis. Semin Liver Dis 2001;21:437-451.

4 Gabele E, Brenner DA, Rippe RA: Liver fibrosis: Signals leading to the amplification of the fibrogenic hepatic stellate cell. Front Biosci 2003;8:d69-77.

5 Marra F: Hepatic stellate cells and the regulation of liver inflammation. J Hepatol 1999;31:1120-1130.

6 Xu L, Hui AY, Albanis E, Arthur MJ, O’Byrne SM, Blaner WS, Mukherjee P, Friedman SL, Eng FJ: Human hepatic stellate cell lines, lx-1 and lx-2: New tools for analysis of hepatic fibrosis. Gut 2005;54:142-151.

7 Ho JH, Tseng KC, Ma WH, Chen KH, Lee OK, Su Y: Thymosin beta-4 upregulates anti-oxidative enzymes and protects human cornea epithelial cells against oxidative damage. Br J Ophthalmol 2008;92:992-997.

-8 Peng Y, Yang H, Wang N, Ouyang Y, Yi Y, Liao L, Shen H, Hu G, Wang Z, Tao L: Fluorofenidone attenuates hepatic fibrosis by suppressing the proliferation and activation of hepatic stellate cells. Am J Physiol Gastrointest Liver Physiol 2014;306:G253-263.

-9 Fang L, Zhan S, Huang C, Cheng X, Lv X, Si H, Li J: Trpm7 channel regulates pdgf-bb-induced proliferation of hepatic stellate cells via pi3k and erk pathways. Toxicol Appl Pharmacol 2013;272:713-725.

10 Barnaeva E, Nadezhda A, Hannappel E, Sjogren MH, Rojkind M: Thymosin beta4 upregulates the expression of hepatocyte growth factor and downregulates the expression of pdgf-beta receptor in human hepatic stellate cells. Ann N Y Acad Sci 2007;1112:154-160.

11 Greenwel P, Dominguez-Rosales JA, Mavi G, Rivas-Estilla AM, Rojkind M: Hydrogen peroxide: A link between acetaldehyde-elicited alpha1(i) collagen gene up-regulation and oxidative stress in mouse hepatic stellate cells. Hepatology 2000;31:109-116.

12 Malinda KM, Goldstein AL, Kleinman HK: Thymosin beta 4 stimulates directional migration of human umbilical vein endothelial cells. FASEB J 1997;11:474-481.

13 Bock-Marquette I, Saxena A, White MD, Dimaio JM, Srivastava D: Thymosin beta4 activates integrin-linked kinase and promotes cardiac cell migration, survival and cardiac repair. Nature 2004;432:466-472.

14 Zuo Y PS, Yang HC, Ma LJ, Fogo AB.: The balance of thymosin $\beta 4$ and its metabolite ac-sdkp modulates activity of profibrotic factors. Modern Pathol 2010;23:Suppl 1.

15 Zuo Y, Chun B, Potthoff SA, Kazi N, Brolin TJ, Orhan D, Yang HC, Ma LJ, Kon V, Myohanen T, Rhaleb NE, Carretero OA, Fogo AB: Thymosin beta 4 and its degradation product, ac-sdkp, are novel reparative factors in renal fibrosis. Kidney Int 2013;84:1166-1175.

16 Reyes-Gordillo K, Shah R, Popratiloff A, Fu S, Hindle A, Brody F, Rojkind M: Thymosin-beta4 (tbeta4) blunts pdgf-dependent phosphorylation and binding of akt to actin in hepatic stellate cells. Am J Pathol 2011;178:2100-2108.

17 Cho JJ, Hocher B, Herbst H, Jia JD, Ruehl M, Hahn EG, Riecken EO, Schuppan D: An oral endothelin-a receptor antagonist blocks collagen synthesis and deposition in advanced rat liver fibrosis. Gastroenterology 2000;118:1169-1178.

18 Boigk G, Stroedter L, Herbst H, Waldschmidt J, Riecken EO, Schuppan D: Silymarin retards collagen accumulation in early and advanced biliary fibrosis secondary to complete bile duct obliteration in rats. Hepatology 1997;26:643-649.

19 Bataille F, Rohrmeier C, Bates R, Weber A, Rieder F, Brenmoehl J, Strauch U, Farkas S, Furst A, Hofstadter F, Scholmerich J, Herfarth H, Rogler G: Evidence for a role of epithelial mesenchymal transition during pathogenesis of fistulae in crohn's disease. Inflamm Bowel Dis 2008;14:1514-1527.

-20 Garg V, Kathiriya IS, Barnes R, Schluterman MK, King IN, Butler CA, Rothrock CR, Eapen RS, Hirayama-Yamada K, Joo K, Matsuoka R, Cohen JC, Srivastava D: Gata4 mutations cause human congenital heart defects and reveal an interaction with tbx5. Nature 2003;424:443-447.

21 Nemolato S, Van Eyken P, Cabras T, Cau F, Fanari MU, Locci A, Fanni D, Gerosa C, Messana I, Castagnola M, Faa G: Expression pattern of thymosin beta 4 in the adult human liver. Eur J Histochem 2011;55:e25.

-22 Shuda M, Kwun HJ, Feng H, Chang Y, Moore PS: Human merkel cell polyomavirus small $t$ antigen is an oncoprotein targeting the 4e-bp1 translation regulator. J Clin Invest 2011;121:3623-3634.

23 Safer D, Elzinga M, Nachmias VT: Thymosin beta 4 and fx, an actin-sequestering peptide, are indistinguishable. J Biol Chem 1991;266:4029-4032.

24 Sanders MC, Goldstein AL, Wang YL: Thymosin beta 4 (fx peptide) is a potent regulator of actin polymerization in living cells. Proc Natl Acad Sci U S A 1992;89:4678-4682. 
25 Conte E, Genovese T, Gili E, Esposito E, Iemmolo M, Fruciano M, Fagone E, Pistorio MP, Crimi N, Cuzzocrea S, Vancheri C: Protective effects of thymosin beta4 in a mouse model of lung fibrosis. Ann N Y Acad Sci 2012;1269:69-73.

26 Rubin BK, Kater AP, Goldstein AL: Thymosin beta4 sequesters actin in cystic fibrosis sputum and decreases sputum cohesivity in vitro. Chest 2006;130:1433-1440.

-27 Koutrafouri V, Leondiadis L, Avgoustakis K, Livaniou E, Czarnecki J, Ithakissios DS, Evangelatos GP: Effect of thymosin peptides on the chick chorioallantoic membrane angiogenesis model. Biochim Biophys Acta 2001;1568:60-66.

-28 Malinda KM, Sidhu GS, Mani H, Banaudha K, Maheshwari RK, Goldstein AL, Kleinman HK: Thymosin beta4 accelerates wound healing. J Invest Dermatol 1999;113:364-368.

29 Badamchian M, Fagarasan MO, Danner RL, Suffredini AF, Damavandy H, Goldstein AL: Thymosin beta(4) reduces lethality and down-regulates inflammatory mediators in endotoxin-induced septic shock. Int Immunopharmacol 2003;3:1225-1233.

-30 Naylor PH, McClure JE, Spangelo BL, Low TL, Goldstein AL: Immunochemical studies on thymosin: Radioimmunoassay of thymosin beta 4. Immunopharmacol 1984;7:9-16.

-31 Inzitari R, Cabras T, Pisano E, Fanali C, Manconi B, Scarano E, Fiorita A, Paludetti G, Manni A, Nemolato S, Faa G, Castagnola M, Messana I: Hplc-esi-ms analysis of oral human fluids reveals that gingival crevicular fluid is the main source of oral thymosins beta(4) and beta(10). J Sep Sci 2009;32:57-63.

-32 Bodendorf S, Born G, Hannappel E: Determination of thymosin beta4 and protein in human wound fluid after abdominal surgery. Ann N Y Acad Sci 2007;1112:418-424.

-33 Ikeda H, Nagoshi S, Ohno A, Yanase M, Maekawa H, Fujiwara K: Activated rat stellate cells express c-met and respond to hepatocyte growth factor to enhance transforming growth factor beta1 expression and DNA synthesis. Biochem Biophys Res Commun 1998;250:769-775.

-34 Kim WH, Matsumoto K, Bessho K, Nakamura T: Growth inhibition and apoptosis in liver myofibroblasts promoted by hepatocyte growth factor leads to resolution from liver cirrhosis. Am J Pathol 2005;166:10171028.

-35 Sanchez-Valle V, Chavez-Tapia NC, Uribe M, Mendez-Sanchez N: Role of oxidative stress and molecular changes in liver fibrosis: A review. Curr Med Chem 2012;19:4850-4860.

-36 Parola M, Robino G: Oxidative stress-related molecules and liver fibrosis. J Hepatol 2001;35:297-306.

-37 Svegliati-Baroni G, Inagaki Y, Rincon-Sanchez AR, Else C, Saccomanno S, Benedetti A, Ramirez F, Rojkind M: Early response of alpha2(i) collagen to acetaldehyde in human hepatic stellate cells is tgf-beta independent. Hepatology 2005;42:343-352.

38 Kumar S, Gupta S: Thymosin beta 4 prevents oxidative stress by targeting antioxidant and anti-apoptotic genes in cardiac fibroblasts. PLoS One 2011;6:e26912.

-39 Lauffenburger DA, Horwitz AF: Cell migration: A physically integrated molecular process. Cell 1996;84:359369.

40 Pollard TD, Borisy GG: Cellular motility driven by assembly and disassembly of actin filaments. Cell 2003;112:453-465.

-41 Fan Y, Gong Y, Ghosh PK, Graham LM, Fox PL: Spatial coordination of actin polymerization and ilk-akt2 activity during endothelial cell migration. Dev Cell 2009;16:661-674.

42 Friedman SL: Liver fibrosis -- from bench to bedside. J Hepatol 2003;38:S38-53. 